\title{
Structural Relaxation of Ultrathin Polymer Films Prepared by the Langmuir-Blodgett Technique. Molecular Weight Effect on the Diffusion of Polymer Segments
}

\author{
Shinzaburo Ito, Kenji Kawano, Michiaki Mabuchi, and Masahide Yамамото* \\ Department of Polymer Chemistry, Graduate School of Engineering, Kyoto University, \\ Sakyo, Kyoto 606-01, Japan
}

(Received October 4, 1995)

\begin{abstract}
Structural relaxation of polymer Langmuir-Blodgett (LB) films has been studied by the energy transfer method, in particular, with respect to the molecular weight $\left(M_{w}\right)$ effect on the diffusion processes. A very high molecular weight poly(vinyl octanal acetal) (PVO) was prepared as the host matrix of the LB multilayers. The low molecular weight PVO's (mono-dispersed in the range of $M_{w}=1.6 \times 10^{4}-2 \times 10^{5}$ ) were labeled with energy donating and accepting probes, and these monolayers were embedded into the high molecular weight PVO layers. The energy transfer measurements provided selectively the diffusion constants $(D)$ of the low molecular weight components in the direction normal to the layer plane. The values of $D$ rapidly decreased with increasing the molecular weight, but leveled out for the higher $M_{w}$ 's than $6 \times 10^{4}$, i.e., they became almost independent of the molecular weight. This striking effect indicates that when the size of single polymer chain becomes larger than the spatial distance examined, the relaxation of the layered structure takes place mainly by the segmental motion of polymer chains from nonequilibrium conformations confined in the two-dimensional plane.

KEY WORDS Polymer Langmuir-Blodgett Film/Energy Transfer Method/Structural Relaxation Diffusion Coefficient / Molecular Weight Effect /
\end{abstract}

Recently, polymer LB films have been investigated extensively because of the characteristic and favorable properties which have not been expected to the conventional $\mathrm{LB}$ films made from long-chain fatty acids. ${ }^{1}$ In particular, the polymeric materials seem to have much better stability of the layered structure compared with the low molecular weight materials. We have been studying the thermal stability of polymer LB films by using the fluorescence method. ${ }^{2,3}$ The energy transfer phenomenon is quite sensitive to the alteration of layer distances in a scale of nanometer, furthermore, it allows in situ observation on the course of structural relaxation. ${ }^{4,5}$ By using this method, the aging effect of the layered structure and the thermal relaxation under elevated temperatures have been investigated. The previous paper of this series reported the diffusion constant of polymer chains in the layered structure and its apparent activation energy $\Delta E$, which was only one third the activation energy of the shift factor measured by mechanical spectroscopy for the bulk film. ${ }^{5,6}$ This result strongly suggested that the thermal relaxation process of thin LB films is governed by the segmental motion of polymer chains.

To confirm this inference, the investigation of the molecular weight effect on the diffusion process is highly desirable. The current study is focused on this point using a combination of an extremely high molecular weight polymer sample and mono-dispersed low molecular weight polymers bearing fluorescent probes.

\section{EXPERIMENTAL}

\section{Materials}

Poly(vinyl octanal acetal) (PVO) was synthesized from poly(vinyl alcohol) (PVA) as described previously. ${ }^{7,8}$ Here we employed two kinds of PVA as the source

${ }^{\dagger}$ To whom all correspondence should be addressed. polymer; one is very high molecular weight PVA $(d p=$ $12,500)$ offered by Prof. Sangen, and the other is relatively low molecular weight PVA $(d p=500)$ purchased from Aldrich. Only the latter PVA (low molecular weight) was labeled with phenanthrene (P: energy donating probe) or anthracene (A: energy accepting probe) by the reaction with the corresponding chromophoric aldehydes (Aldrich). The chemical structure is shown in Chart 1 and the characteristics of the obtained polymers are listed in Table I. These PVO's were named as PVO-H (high molecular weight), PVO-LP and PVO-LA (low molecular weight labeled with $\mathrm{P}$ and $\mathrm{A}$, respectively). PVO-LP and PVO-LA were fractionated by GPC which was calibrated by the molecular weight determined by

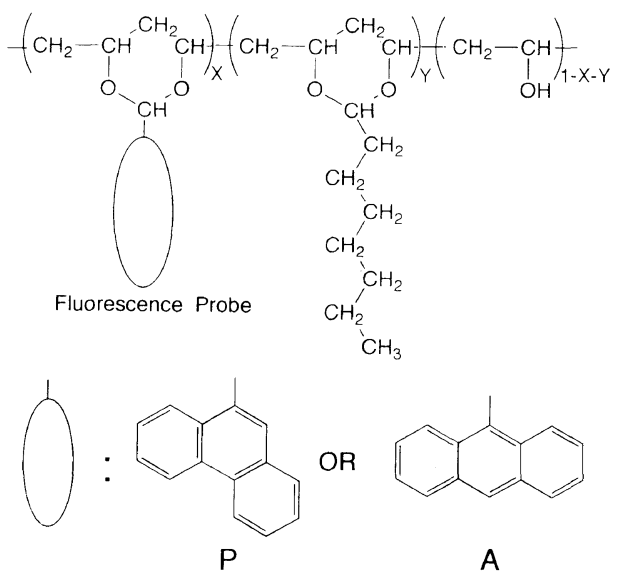

Table I. Degree of polymerization of PVA and compositions of synthesized PVO polymers

\begin{tabular}{lcccc}
\hline Sample & $d p$ of PVA & $\begin{array}{c}\text { Alkanal } \\
\text { unit } / \%\end{array}$ & $\begin{array}{c}\text { Chromophoric } \\
\text { unit } / \%\end{array}$ & $\begin{array}{c}\text { Hydroxy } \\
\text { unit } / \%\end{array}$ \\
\hline PVO-H & 12500 & 71 & 0 & 29 \\
PVO-LP & $300-500$ & 61.1 & 8.7 & 30.2 \\
PVO-LA & $300-500$ & 56.3 & 6.5 & 37.2 \\
\hline
\end{tabular}


light scattering measurements (Chromatix model KMX6). The GPC chromatograms of these polymers are shown in Figure 1. As the result, we obtained five fractions of PVO-LP and PVO-LA having molecular weight in the range of $1.6 \times 10^{4}$ to $2 \times 10^{5}$ with narrow dispersion (see Table II). As to PVO-H, the exact value of molecular weight could not be determined because it was too high, being out of the discernible range of our GPC column. Therefore, the value was estimated from the molecular weight of PVA and the content of alkyl substituents determined by elementary analysis. The obtained value is more than $10^{6}$ which is large enough compared with those of PVO-L samples.

\section{Sample Preparation}

Polymer LB films were prepared on a non-fluorescent quartz plate. The details have already been described previously. ${ }^{8}$ Figure 2 depicts the layer structure of LB films for measuring the molecular weight effect. The sample films were built-up in the following sequence with Y-type deposition: (1)four layers of PVO-H for the
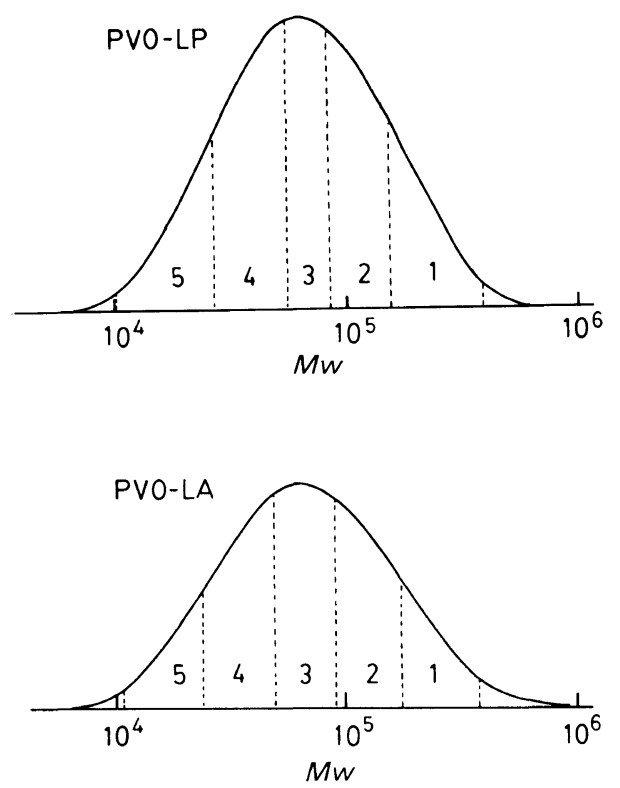

Figure 1. GPC chromatograms of fluorescent probe labeled PVO's and the fractionation into five ranges of molecular weight.

Table II. Molecular weights, dispersions, and surface pressures at the LB deposition of chromophoric PVO polymers

\begin{tabular}{ccccc}
\hline Sample & $M_{w} / 10^{4}$ & $M_{n} / 10^{4}$ & $M_{w} / M_{n}$ & $\begin{array}{c}\text { Surface pressure } \\
/ \mathrm{mN} \mathrm{m}^{-1}\end{array}$ \\
\hline PVO-LP1 & 18.8 & 17.3 & 1.09 & 18 \\
PVO-LP2 & 10.5 & 9.73 & 1.08 & 17 \\
PVO-LP3 & 6.12 & 5.60 & 1.09 & 19 \\
PVO-LP4 & 3.48 & 3.19 & 1.09 & 15 \\
PVO-LP5 & 1.82 & 1.64 & 1.11 & 15 \\
\hline PVO-LA1 & 20.2 & 18.4 & 1.10 & 20 \\
PVO-LA2 & 10.6 & 9.67 & 1.10 & 20 \\
PVO-LA3 & 5.96 & 5.49 & 1.09 & 18 \\
PVO-LA4 & 3.03 & 2.79 & 1.09 & 16 \\
PVO-LA5 & 1.64 & 1.53 & 1.07 & \\
\hline PVO-H & $(112)^{\mathrm{a}}$ & & &
\end{tabular}

${ }^{a}$ calculated value from the degree of polymerization of PVA and elementary analysis of PVO. precoating layers, (2) two layers of PVO-LP for the energy donating labeled layers, (3) four layers of PVO-H for the spacer, (4) two layers of PVO-LA for the energy accepting labeled layers, and finally (5) six layers of PVO-H again for the protecting layers. Therefore, total number of layers is 18 , i.e., the whole thickness of LB films is $18 \mathrm{~nm}$ because the thickness of each layer is known to be $1 \mathrm{~nm}$ by ellipsometry using the refractive index of PVO: $1.48 .^{7}$ It should be noted that the labeled PVO-LP and PVO-LA layers are separated with four layers of PVO-H whose thickness is only $4 \mathrm{~nm}$. This distance was chosen taking account of the Förster radius of the P-A pair: $2.12 \mathrm{~nm}$. Both outsides of labeled films were covered with PVO-H layers. In this study, the examined layers were embedded into the high molecular weight PVO layers. This layer configuration allows us to observe selectively the diffusion of the low molecular weight components in the LB films.

\section{Measurements}

Fluorescence spectra were recorded by a Hitachi 850 fluorescence spectrophotometer equipped with a thermoregulated sample chamber. The $\mathrm{P}$ chromophores were selectively excited with $298 \mathrm{~nm}$ light. The emission intensity $I_{\mathrm{P}}$ at $350 \mathrm{~nm}$ from $\mathrm{P}$ and the intensity $I_{\mathrm{A}}$ at $438 \mathrm{~nm}$ from $\mathrm{A}$ were used for calculation of the energy transfer efficiency. Although the details of energy transfer analysis have been described previously, ${ }^{5,6}$ the principle should be noted briefly for the sake of further discussion.

The diffusion of polymer segments in the LB film results in a distribution of chromophores in the direction normal to the film plane. We assumed a Gaussian distribution of chromophores;

$$
C(x)=\left(C_{0} /\left(2 \pi \sigma^{2}\right)^{1 / 2}\right) \exp \left(-x^{2} / 2 \sigma^{2}\right)
$$

where $C(x)$ is the concentration of chromophores at the displacement $x, C_{0}$ is related to the total number of chromophores, and $\sigma^{2}$ is the variance of distribution. The use of Gaussian distribution is one of the approximations but seems to be the most reasonable distribution function for expressing the diffusion process. The numerical calculation of energy transfer efficiency under a given variance $\sigma^{2}$, was performed by a computer. Consequently, the chromophore distribution could be determined by comparison of the energy transfer efficiency experimentally obtained with the theoretical one.

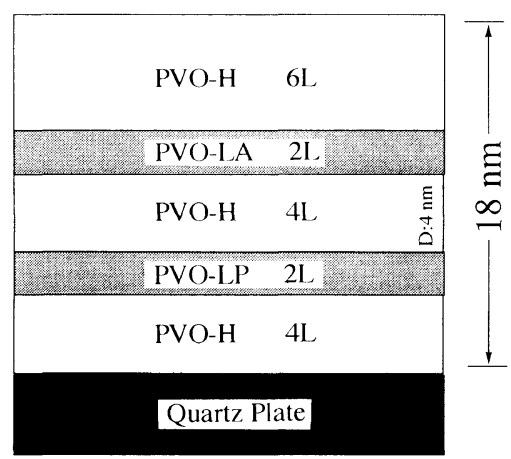

Figure 2. Schematic illustration of LB multilayers for the measurements of energy transfer efficiency. 
The change of transfer efficiencies with time indicates the alteration of $\sigma^{2}$, which was fitted to the theoretical prediction of the Fickian diffusion model:

$$
\sigma^{2}=2 D t+\sigma_{0}^{2}
$$

where $D$ is the diffusion constant of polymer segments and $\sigma_{0}^{2}$ is the initial dispersion due to the Y-type deposition (double layer, $2 \mathrm{~nm}$ thick) and also due to a little disordering at the deposition process. Therefore, the fitting procedure allowed us to evaluate the diffusion constant in the polymer LB films.

\section{RESULTS AND DISCUSSION}

Figure 3 depicts a typical example of time evolution of the energy transfer efficiencies at 25,30 , and $40^{\circ} \mathrm{C}$ for the PVO-LP3 and LA3 in PVO-H layers. These temperatures were chosen to be close or slightly higher than the glass transition temperature of $\mathrm{PVO}, 25^{\circ} \mathrm{C}$, measured by DSC. Obviously the data at higher temperatures show the faster increase of energy transfer efficiency, yielding larger diffusion constants $D$ 's as the result of the fitting procedure. The solid lines in Figure 3 represent the theoretical values using the best fit parameters in eq 2 .

Similar to the temperature dependence, the lower molecular weight samples provided the faster increase of energy transfer efficiency at a constant temperature. The whole data at a couple of temperatures are given in Figure 4, where the diffusion constants obtained are plotted against the molecular weight in the logarithmic scale.

As expected, the larger the molecular weight of samples, the smaller the diffusion constants were observed. However, the clear effect of the molecular weight was seen only in the left-hand side of Figure 4, i.e., in the low molecular weight region. The slope of solid lines in Figure 4 was found to be close to -2 , i.e., the diffusion constant decreases with the inverse second power of the molecular weight:

$$
D \propto M_{w}^{-2} .
$$

Such tendency has been often observed in various

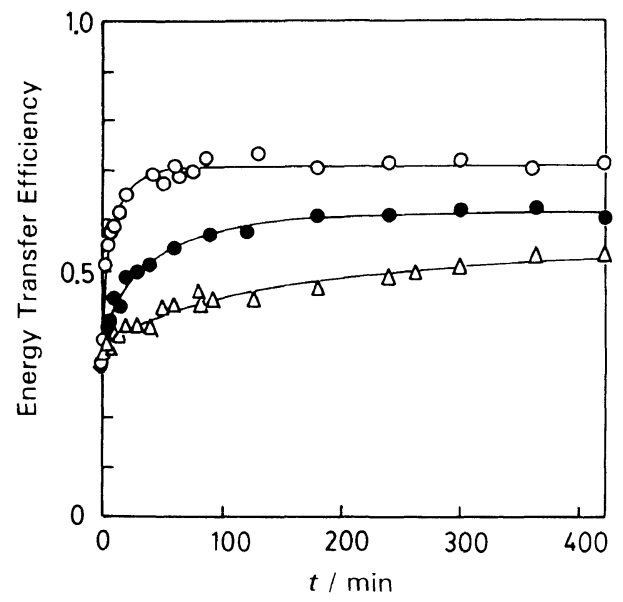

Figure 3. Time evolution of energy transfer efficiencies for the PVO-LP3-PVO-LA3 film at different temperatures: (O) $40^{\circ} \mathrm{C}$; (O) $30 \mathrm{C} ;(\triangle) 25 \mathrm{C}$. Solid lines represent the calculation curves fitted with parameters in eq 2 , e.g., $D=4.54 \times 10^{-17} \mathrm{~cm}^{2} \mathrm{~s}^{-1}, \sigma_{0}=2.3 \mathrm{~nm}$ at $30^{\circ} \mathrm{C}$. polymer bulk systems. For example, Wool et al. have studied diffusion phenomena at the interface of two kinds of polystyrene films (protonated and deuterated) by secondary ion mass spectroscopy. ${ }^{9}$ They reported molecular weight dependence on self-diffusion coefficients observed at long diffusion times,

$$
D \propto M_{w}^{-2.1 \pm 0.2}
$$

with good correlation coefficients close to unity. The de Gennes reptation model predicts the relationship of $D \propto M_{w}^{-2}$ for entangled chains at diffusion times longer than the reptation time $\tau$ at which the whole chain escapes from the initial configuration. Winnik et al. also have reported similar correlation between the molecular weight and polymer diffusion at the interfaces of latex films using the energy transfer technique. ${ }^{10}$ The decrease in $D$ could be accounted for approximately as $D \propto M_{w}^{-2.3}$. Therefore, the result we observed in Figure 4 indicates that the center of mass motion of polymer chains probably dominates the diffusion process in the lower molecular weight region.

For the high molecular weight samples, however, one could see very little molecular weight dependence. It is very hard to explain this behavior by the previous discussion based on the mass diffusion of polymer chain; another relaxation mode must be taken into account. As reported previously, we observed quite small apparent activation energy $\Delta E$ of the diffusion constant, compared with that observed for the bulk film. ${ }^{6}$ This result strongly suggests that the structural relaxation process of thin LB films is governed by the segmental motion of polymer chains. The previous experiments were performed by using high molecular weight samples with polydispersity, ca. $M_{w}=4 \times 10^{5}$, which is much larger than the critical molecular weight (the inflection point in Figure 4) appeared in the current study. Therefore, it is worthwhile to examine $\Delta E$ at various molecular weights. Figure 5 depicts the Arrhenius plot of $D$, showing the gradual decrease of $\Delta E$ with increasing the molecular weight: $190 \mathrm{~kJ} \mathrm{~mol}^{-1}$ for LP5-LA5 $\left(M_{w}=c a\right.$. $\left.2 \times 10^{4}\right)$ and $120 \mathrm{~kJ} \mathrm{~mol}^{-1}$ for LP3-LA3 $\left(M_{\mathrm{w}}=c a\right.$. $6 \times 10^{4}$ ). This indicates that the relaxation mechanism of LB films tends to alter from the whole chain motions to the segmental motions of local chains.

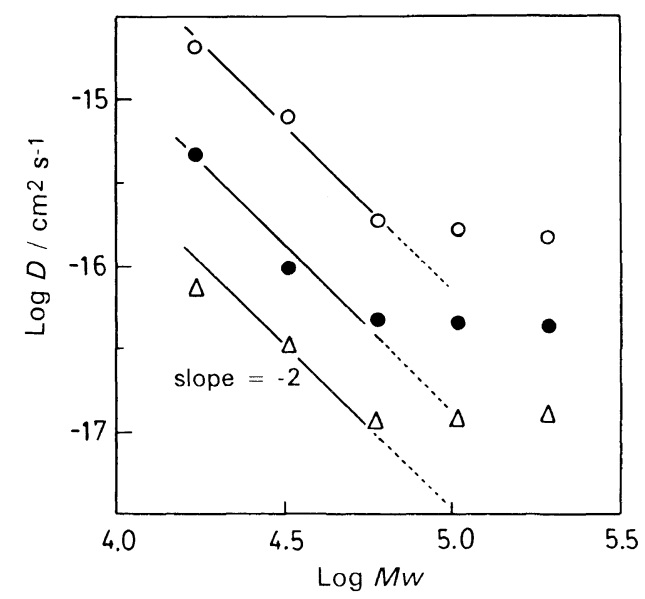

Figure 4. Molecular weight dependence of diffusion constants at different temperatures:

(○) $40 \% \mathrm{C} ;\left(\mathrm{O}^{\circ} 30^{\circ} \mathrm{C} ;(\triangle) 25^{\circ} \mathrm{C}\right.$. 


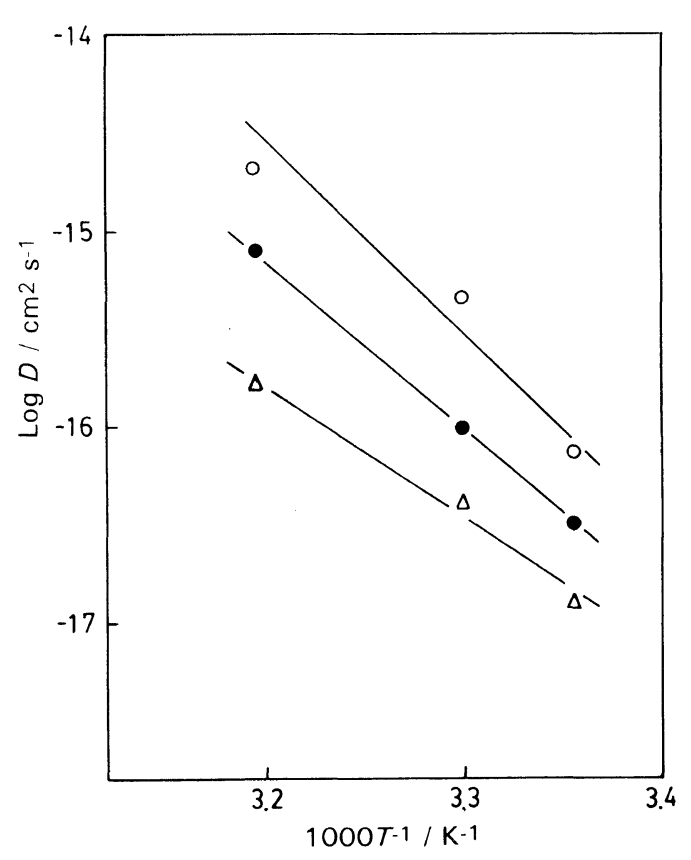

Figure 5. Arrhenius plot of diffusion constants for: (O) PVO-

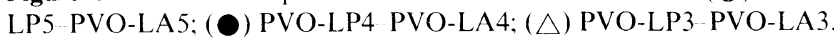

Table III. Radius of a polymer chain confined in a two-dimensional plane

\begin{tabular}{ccc} 
Sample & $M_{w} / 10^{4}$ & $R_{2 \mathrm{D}} / \mathrm{nm}$ \\
\hline PVO-LP1 & 18.8 & 10.6 \\
PVO-LP2 & 10.5 & 7.8 \\
PVO-LP3 & 6.12 & 5.9 \\
PVO-LP4 & 3.48 & 4.3 \\
PVO-LP5 & 1.82 & 3.2
\end{tabular}

Let us consider the size of single polymer chain which is confined in a two-dimensional plane on the process of LB deposition; a dilute polymer solution was spread on a water surface, then the polymer chains adsorbed on the surface were compressed while keeping the twodimensional conformation. Although there is no information about two-dimensional expansion factor of the chain conformation, one can easily estimate the minimum radius of circle occupied by a single polymer chain at the compressed form. Table III is the list of radius for various molecular weight samples, estimated by the surface area at the deposition point. Figure 6 is a schematic illustration of the present system. Even at the compressed form, a polymer chain occupies a large area; the radius of the high molecular weight sample exceeds the distance of separation $(4 \mathrm{~nm})$ between the energy donating and accepting layers. Therefore, segmental motions of a part of long chain is enough to disorder the whole layer structure, consequently, the diffusion rate observed becomes less dependent on the molecular weight.

Theoretically and experimentally, local chain motions in a polymer bulk and at their interfaces have been extensively discussed. ${ }^{11-15}$ Zang et al. analyzed chain dynamics at times shorter than the reptation time $\tau$ using both the Rouse and the reptation relaxation models. ${ }^{9,13}$ Their important conclusion is that the interdiffusion at $t<\tau$ involves the relaxation of the nonequilibrium chain conformation and the segmental motion tends to

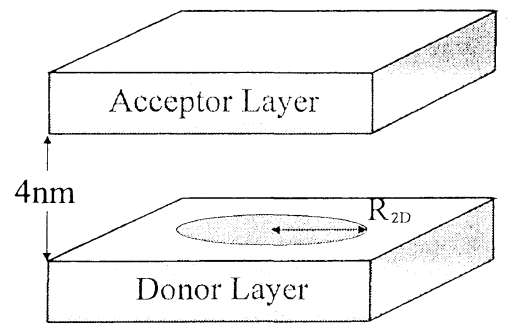

Figure 6. Schematic illustration of the layer configuration and molecular size of a polymer chain.

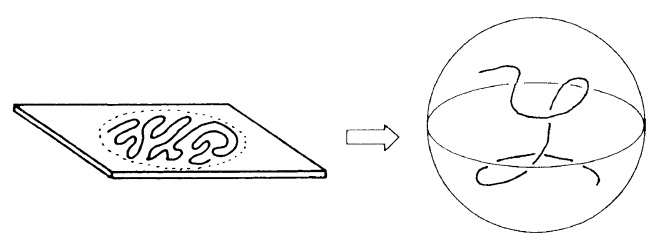

2D

$3 \mathrm{D}$

Figure 7. Illustration of entropy relaxation from a compressed plane conformation to a 3-dimensional randam-coil conformation.

dominate the diffusion process. Although the discussion was done mainly with respect to the time range of observation, this is also the case for the spatial dimension of observation; the smaller the size of observation, the larger the contribution of segmental motions is. Therefore, when the diffusion distance becomes shorter than the radius of gyration of a polymer chain, the local motions come up as the rate-determining factor of the structural relaxation, and the observed diffusivity becomes apparent one since it is not the value for the true center of mass motion.

Considering local motions of a polymer chain, one should care whether the chain conformation at the initial stage is in an equilibrium or not. Obviously the polymer chain deposited by the LB method takes a nonequilibrium conformation imposed by the adsorption force of the water surface. As illustrated in Figure 7, the two-dimensional form confined in a plane must be released and expanded to a three-dimensionally equilibrium form when the chain movement is allowed by thermal agitation. Such a relaxation from a nonequilibrium state is likely to have a smaller activation energy and a faster relaxation response compared with those for equilibrium chains.

\section{CONCLUSION}

The structural relaxation of polymer LB films was investigated by the energy transfer method. The results indicated that the relaxation mechanism should be discussed taking into account both the spatial distance examined and the molecular size of a polymer chain. For the smaller size of chains compared with the observed distance, the relaxation proceeded by the center of mass motion of chains in the polymer matrix, but for the larger molecules the relaxation was governed by the local segmental motion of polymer chains from nonequilibrium conformations imposed in a two-dimensional LB film. This resulted in a small activation energy and larger diffusivity than the equilibrium chains. We may call this phenomenon an "entropy relaxation". 
Most of polymer LB films studied so far have been utilized in the high molecular weight region. Therefore, an important key for realizing thermally stable nanomaterials is to fix the segmental motion of the polymer chains.

Acknowledgments. We thank Prof. O. Sangen and Dr. T. Yamamoto of Himeji Institute of Technology for their kind gift of high molecular weight PVA. For the light scattering measurements we thank Prof. T. Matsumoto of Kyoto University. This work was partly supported by a Grant-in-Aid for Scientific Research (No. 06651050) from the Ministry of Education, Science, and Culture of Japan.

\section{REFERENCES}

1. International Conferences on Langmuir-Blodgett Films; Paris, 1991. Proceedings published in: Thin Solid Films, 210/211 (1992) Quebec, 1993. Proceedings published in: Thin Solid Films, 242
244 (1994).

2. S. Ohmori, S. Ito, and M. Yamamoto, Macromolecules, 24, 2377 (1991).

3. T. Ueno, S. Ito, S. Ohmori, Y. Onogi, and M. Yamamoto, Macromolecules, 25, 7150 (1992).

4. S. Ito, T. Ueno, and M. Yamamoto, Thin Solid Films, 211, 614 (1992).

5. T. Hayashi, T. Okuyama, S. Ito, and M. Yamamoto, Macromolecules, 27, 2270 (1994).

6. M. Yamamoto, K. Kawano, T. Okuyama, T. Hayashi, and S. Ito, Proc. Jpn. Acad., Ser. B, 70, 121 (1994).

7. M. Watanabe, Y. Kosaka, K. Sanui, N. Ogata, K. Oguchi, and T. Yoden, Macromolecules, 21, 2997 (1988).

8. S. Ohmori, S. Ito, and M. Yamamoto, Macromolecules, 23, 4047 (1990).

9. S. J. Whitlow and R. P. Wool, Macromolecules, 24, 5926 (1991)

10. Y. Wang and M. A. Winnik, J. Phys. Chem., 97, 2507 (1993).

11. Y. H. Kim and R. P. Wool, Macromolecules, 16, 1115 (1983).

12. F. Brochard, J. Jouffroy, and P. Levinson, Macromolecules, 16, 1638 (1983).

13. H. Zhang and R. P. Wool, Macromolecules, 22, 3018 (1989).

14. S. Yukioka, K. Nagato, and T. Inoue, Polymer, 33, 1171 (1992)

15. H. Eklind and T. Hjertberg, Macromolecules, 26, 5844 (1993). 